\title{
Process Development for the Identification of Novel Microbodies against NSCLC Related Targets
}

\author{
Bonny Gaby Lui, Joycelyn Wüstehube-Lausch, Hans-Ulrich Schmoldt, Matin Daneschdar, Ugur Sahin \\ BioNTech AG \\ An der Goldgrube 12, 55131 Mainz, Germany \\ Gaby.Lui@biontech.de
}

\section{Extended Abstract}

Protein scaffolds are a new generation of affinity proteins specialized to complement the antibody and antibody derivatives for therapeutic and diagnostic applications [1]. Cystine-knot miniproteins (Microbodies) represent an alternative protein scaffold with certain drug-desired properties such as high target affinity and specificity, in particular stability, solubility and pharmacokinetic behavior of the binding protein [2,3]. Additionally this small peptidic molecule with a simple architecture facilitates an easy straight-forward chemical production and the construction of multi-functional fusion molecules [4]. Site-directed conjugation of a cystine-knot miniprotein with a radionuclide already showed specific targeting of U87MG tumor-bearing mice [5]. Tumor specific uptake of an imaging agent offers a promising benefit for in vivo visualization to facilitate surgically cancer removal as well as cancer staging [4]. All in all cystine-knot miniproteins is an ideally suitable scaffold for in vivo diagnostic imaging tools. Moreover this protein can be used as scaffold for delivery of therapeutic payloads and functionalized nanoparticle drugs $[4,6]$.

Microbody ${ }^{\circledR}$ owing a remarkable stability and an extraordinary tolerance to sequence variation, which enables the construction of combinatorial libraries [7]. Based on an open chain variant of the squash trypsin inhibitor MCoTI-II found in gac fruit (Momordica cochinchinensis) combinatorial phage libraries was designed to allow the identification of high affine binders against respective target proteins $[8,9]$. The phage display technique provides a powerful tool for the screening of variants in a high throughput manner to select protein-protein interacting binders [10]. In this work three novel Microbody ${ }^{\circledR}$ phage libraries with certain randomized amino acids in defined loop positions could be successfully generated. The analysis of those generated libraries on phage level indicated the functional surface presentation of Microbodies. Furthermore the development of a cell-based screening process enables the targeting of membrane embedded proteins in their native conformation.

For Microbody ${ }^{\circledR}$-based applications plasma membrane proteins or extracellular located proteins can be addressed. Based on mRNA expression data a transmembrane cell adhesion protein was selected as a non-small cell lung cancer associated target protein. The target is substantially expressed on lung squamous cancer and shows only minimal background expression in thoracic region of healthy tissues. In order to validate the candidate as a NSCLC associated target on protein level, western blot analysis with lung cancer patient tissues were performed. A correlation of mRNA expression data and protein expression analysis could be observed and confirmed the upregulation in NSCLC patient samples. Currently, different cell-based screening experiments of Microbody ${ }^{\circledR}$ phage libraries against the target are performed in order to identify novel Microbody ${ }^{\circledR}$ ligands for diagnostic and therapeutic applications.

\section{References}

[1] M. Gebauer et al., "Engineered protein scaffolds as next-generation antibody therapeutics." Current opinion in chemical biology," Curr. opin. in chem. biol., vol. 13, no. 3, pp. 245-255, 2009.

[2] T. Wurch et al., "Novel protein scaffolds as emerging therapeutic proteins: from discovery to clinical proof-ofconcept," Trends Biotechnol., vol. 30, no. 11, pp. 575-582, 2012.

[3] L.A. Stern et al., "Alternative non-antibody protein scaffolds for molecular imaging of cancer," Curr. opin. ohem. Eng., vol. 2, no. 4, pp. 425-432, 2013. 
[4[ H. Kolmar, "Engineered cystine-knot miniproteins for diagnostic applications," vol. 10, no. 3, pp. 361-368, Expert. Rev. Mol. Diagn., 2010.

[5] L. Jiang et al., "A Radiofluorinated Divalent Cystine Knot Peptide for Tumor PET Imaging," Mol. Pharm., vol. 11, no. 11, pp. 2885-2892, 2014.

[6] S. A. Ackerman et al., "Cystine-knot peptides: emerging tools for cancer imaging and therapy." Expert review of proteomics," Trends Biotechnol., vol. 11, no. 5, pp. 561-572, 2014.

[7] R. H. Kimura et al, "Pharmacokinetically stabilized cystine knot peptides that bind alpha-v-beta-6 integrin with singledigit nanomolar affinities for detection of pancreatic cancer," Cancer Res. vol. 18, no. 3, pp. 839-849, 2009.

[8] O. Avrutina et al., "Trypsin inhibition by macrocyclic and open-chain variants of the squash inhibitor MCoTI-II," Biol. Chem., vol. 386, no. 12, pp. 1301-1306, 2005.

[9] R. H. Kimura et al., "Pharmacokinetically stabilized cystine knot peptides that bind alpha-v-beta-6 integrin with singledigit nanomolar affinities for detection of pancreatic cancer," Clin. Cancer Res., vol. 18, no. 3, pp. 839-849, 2012.

[10] G. P. Smith, "Filamentous fusion phage: novel expression vectors that display cloned antigens on the virion surface," Science, vol. 228, no.4705, pp. 1315-1317, 1985. 